\title{
Assessment of antibiotic prophylaxis prescribing patterns for TURP: A need for Canadian guidelines?
}

\author{
Keith A. Lawson, MD; Jan K. Rudzinski, MD; Ingrid Vicas, MD; Kevin V. Carlson, MD, FRCSC ${ }^{*}$ \\ *Southern Alberta Institute of Urology, Division of Urology, Department of Surgery, University of Calgary, Calgary, AB; †Physician Learning Program, University of Calgary, Calgary, AB
}

See related article on page e 543 .

Cite as: Can Urol Assoc J 2013;7(7-8):e530-6. http://dx.doi.org/10.5489/cuaj.205

Published online August 19, 2013.

\section{Abstract}

Background: While antibiotic prophylaxis is recommended to all patients undergoing transurethral resection of prostate (TURP), little data exist regarding prescribing patterns of urologists prior to this procedure. Here, we sought to determine real-world antibiotic prophylaxis prescribing patterns at a high volume Canadian institution and determine compliance rates to recommendations put forth by the American Urological Association's (AUA) Best Practice Statement (BPS) on antimicrobial prophylaxis.

Methods: A retrospective chart review of 488 patients undergoing TURP was conducted. Electronic medical records were reviewed to determine antibiotics prescribed 3 hours preoperatively and 24 hours postoperatively. For patients without a catheter, compliance was defined as those receiving an antibiotic prior to TURP. In patients with an indwelling catheter, compliance was defined as those receiving antibiotics from two different classes prior to surgery. Results: Overall, a total of 30 antibiotic regimens were utilized. The most common single antibiotic regimens prescribed were ciprofloxacin $(32 \%)$, cefazolin $(25 \%)$ and gentamicin $(3 \%)$. In those patients with indwelling Foley catheters prior to TURP, a significant increase in gentamicin, as well as combination antibiotic regimens, was noted. The compliance rate with the AUA BPS in patients without a preoperative catheter was $81 \%$, while the compliance rate for patients with an indwelling catheter prior to TURP was 37\%.

Interpretation: Collectively, our results demonstrate that prescribing patterns vary significantly prior to TURP, with compliance to AUA BPS being lower than anticipated. Overall, these results support educational efforts in this area, and the development of Canadian recommendations to improve uptake by practicing urologists.

\section{Introduction}

Transurethral resection of prostate (TURP) is a clean contaminated surgical procedure for management of benign pros- tatic hyperplasia (BPH). ${ }^{1-3}$ The postoperative complications of TURP include hematuria, dysuria, fever and bacteriuria. ${ }^{4-6}$ Urological manipulation with TURP instrumentation is a recognized cause of post-TURP urinary tract infections (UTI), as well as possible septicemia. ${ }^{4,7-9}$ The reported incidence of postoperative UTI is between $6 \%$ and $60 \%{ }^{4,6,10}$ Recognized sources of infection include urethral bacterial flora, urethral catheters, intra-operative or postoperative surgical contamination, and infection transferred from a distant sites. ${ }^{8,11-15}$ Moreover, factors which can contribute to increase in risk of post-TURP UTI include presence of preoperative infected urine, indwelling catheter, advanced age, anatomic abnormalities of the urinary tract, smoking, immune-suppression and prolonged hospitalization. 5,13,16,17

There is strong evidence supporting the use of prophylactic antibiotics prior to TURP procedure. In a meta-analysis of 32 randomized controlled trials (RCT) including 4260 patients, antibacterial prophylaxis prior to TURP significantly reduced the incidence of bacteriuria and clinical sepsis. ${ }^{6}$ A similar meta-analysis with 28 trials and 4694 patients has also shown the benefit of preoperative antibiotic use in decreasing incidence of post-TURP bacteriuria, high fever, bacteremia and additional antibiotic treatment. ${ }^{4}$ Clinical efficacy was shown for antibiotic classes including fluoroquionolones, third generation cephalosporins, aminoglycosides and trimethroprim-sulfamethoxazole (TMP-SMX). ${ }^{18-25}$

Based on the available evidence, the 2008 American Urology Association (AUA) Best Practice Statements (BPS) on Urologic Surgery Antimicrobial Prophylaxis recommends use of prophylactic antibiotics for all patients undergoing TURP. ${ }^{1}$ The recommended first-line agents are fluoroquinolones or TMP-SMX, with alternatives including aminoglycosides alone or with ampicillin, cephalosporins or amoxicillin/clavulanate $\leq 24$ hours prior to TURP. In cases where an external urinary catheter is present or placed at the time of procedure, the AUA BPS recommends additional antibiotic treatment for patients with risk factors. ${ }^{1}$

In contrast to the AUA, there are no standardized Canadian consensus statements on the use of prophylactic antibiotics for patients undergoing TURP. Moreover, there is a signifi- 


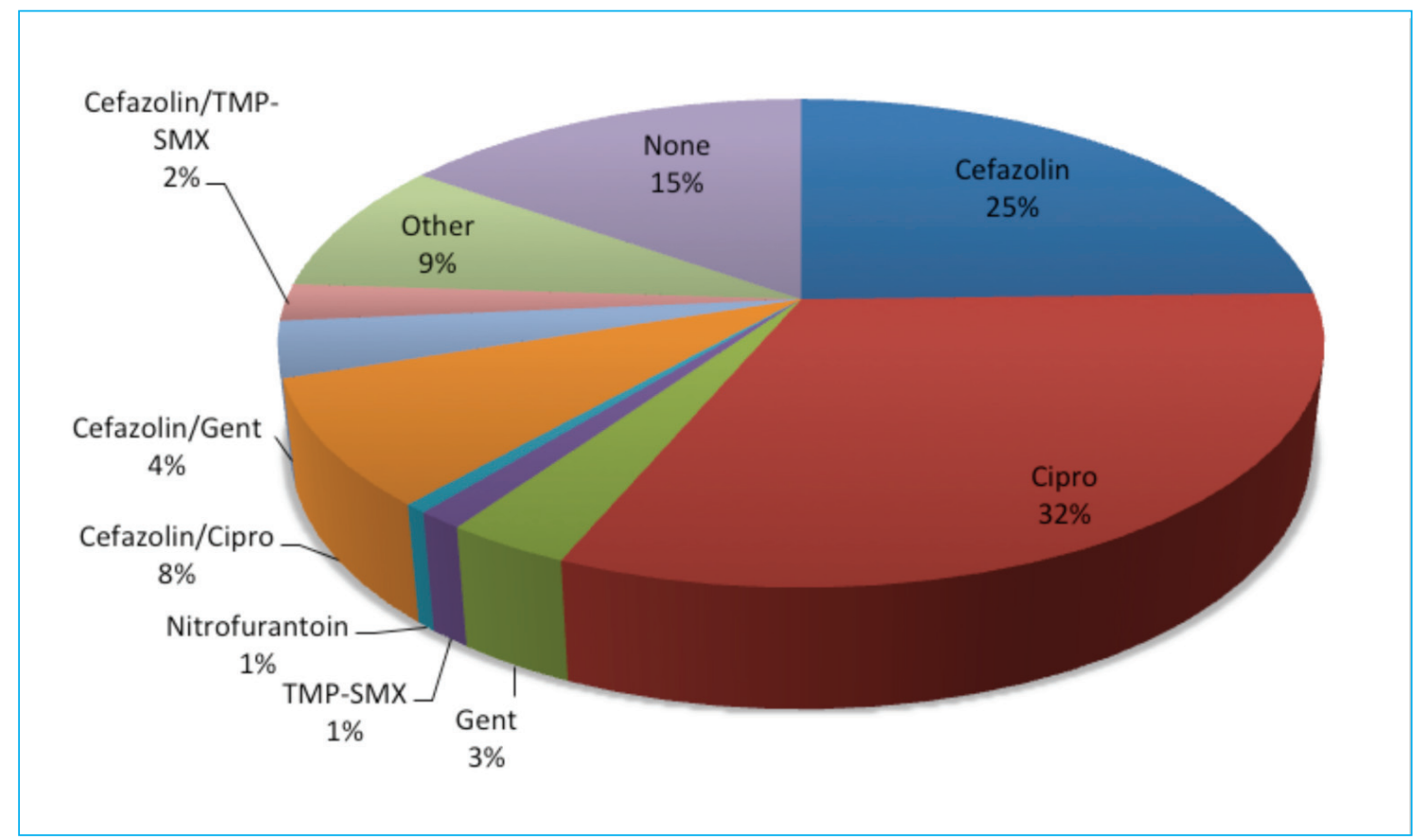

Fig. 1. Pie chart depicting antibacterial prophylaxis for all TURP patients. Antibiotics given 3 hours preoperatively to 24 hours postoperatively were tabulated. Percentages of total patients receiving given antibiotic regimen are depicted. Septra: Septra DS; Cipro: ciprofloxacin; Gent: gentamicin; TMP-SMX: trimethroprim-sulfamethoxazole.

cant lack of literature regarding the actual prescribing patterns of prophylactic antibiotics for this procedure. Thus, as consensus among Canadian urologists regarding this issue is unknown, we sought to characterize prescribing patterns of prophylactic antibiotics prior to TURP in real-world clinical practice in a large Canadian centre. Additionally, we compared our results to the recommendations put forth by the AUA BPS to measure compliance with this document to gain insight into its influence and applicability to our healthcare system. This study is part of a larger Physician Learning Program undertaken by the Division of Urology at the University of Calgary, Calgary, Alberta.

\section{Methods}

We conducted a retrospective chart review and electronic health record review of 488 patients who underwent either standard TURP, transurethral resection in saline (TURIS; Olympus, Center Valley, PA), or GreenLight laser (American Medical Systems; Minnetonka, MN) photovapourization of prostate (PVP) between September 2009 and June 2010 in Calgary, Alberta, Canada (herein referred to as the "TURP" group). Informed consent was obtained by all participating urologists, which included all members of the Division of Urology at the University of Calgary. Ethics approval for the study was granted by the Chair of Conjoint Health Research Ethics Board of the University of Calgary.

Sunrise Clinical Manager (SCM), an electronic health records system which includes medical orders, medication administration records, as well as diagnostic imaging and laboratory results, was reviewed to obtain all antibiotics prescribed 3 hours prior to surgery and 24 hours post-surgery. This was supplemented by manual chart review. Additional variables collected included patient demographics, urgency of procedure and preoperative catheter status.

Patients were categorized according to preoperative catheter status and analyzed for agent(s) and administration time of the pre-TURP antibiotic regimen utilized. Compliance with the 2008 AUA BPS was then determined. For patients without a catheter, compliance was defined as those receiving an antibiotic prior to TURP. In patients with an externalized indwelling urinary catheter preoperatively, compliance was defined as those receiving antibiotics from two different classes prior to surgery. This represents our interpretation of the AUA BPS which states, "In cases where an external urinary catheter is present prior to or is placed at the time of the procedure, additional antimicrobial treatment $(\leq 24$ hours) is recommended in patients with risk factors." Risk factors listed include the presence of an externalized urinary catheter.

\section{Results}

For the 9-month study period, 488 patients were identified. Data analysis on prescribing patterns was conducted on all 488 patients (Table 1, Fig. 1) The most common single antibiotic regimens prescribed were ciprofloxacin $(32 \%)$, cefazolin $(25 \%)$ and gentamicin $(3 \%)$, respectively. The 


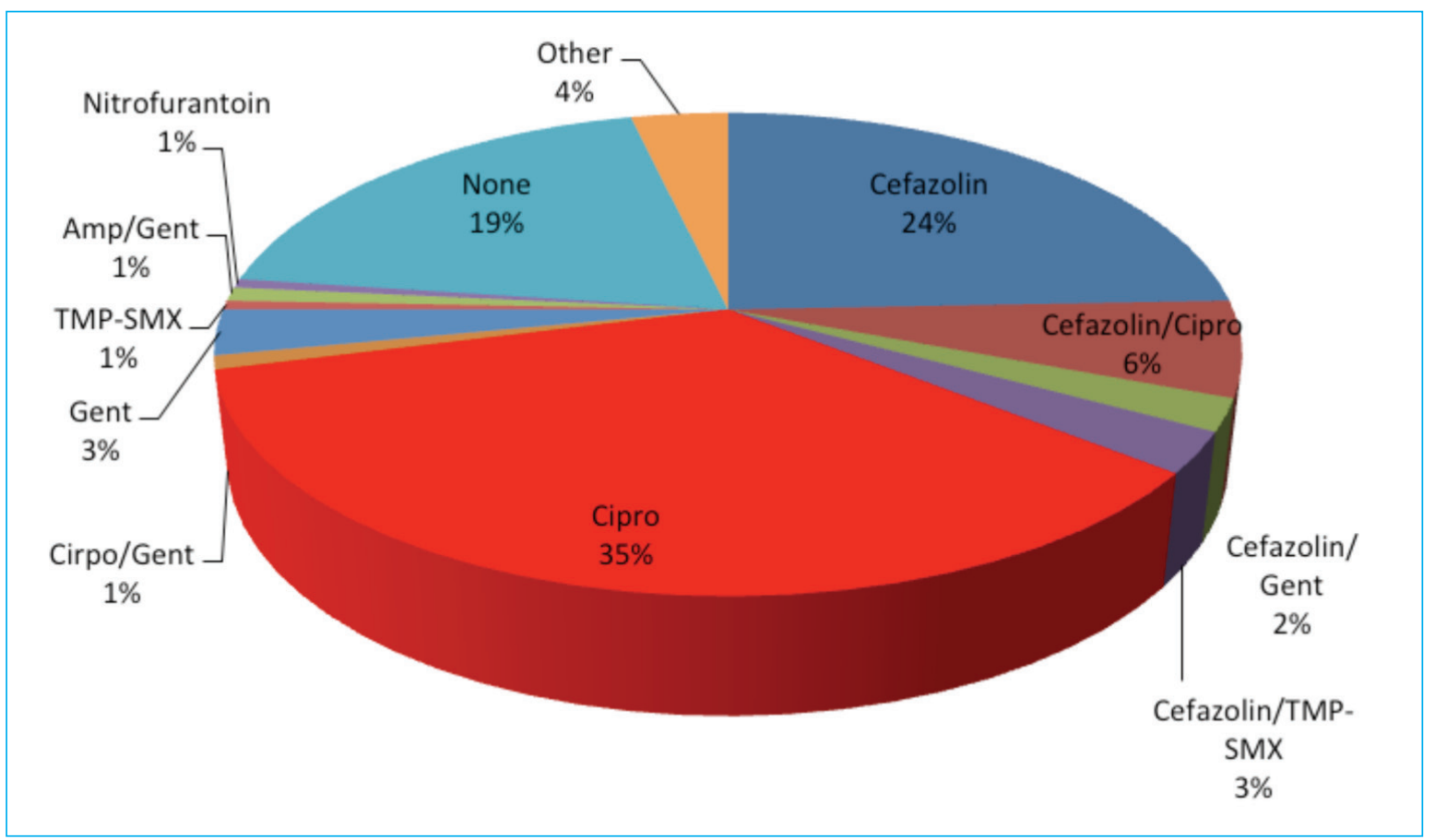

Fig. 2. Pie chart depicting antibacterial prophylaxis for patient without indwelling catheter. Antibiotics given 3 hours preoperatively to 24 hours postoperatively were tabulated. Percentages of total patients without preoperative indwelling catheters receiving a given antibiotic regimen are depicted. Septra: Septra DS; Cipro: ciprofloxacin; Gent: gentamicin; TMP-SMX: trimethroprim-sulfamethoxazole.

most common combinations of antibiotics prescribed were cefazolin with ciprofloxacin (8\%), cefazolin with TMP-SMX $(2 \%)$ and cefazolin with gentamicin (4\%). Of the total number of patients, $74(15 \%)$ received no antibiotic prophylaxis prior to TURP.

A total of $328(67 \%)$ patients had no indwelling catheter prior to surgery. When looking at the antibiotic regimens (Table 2, Fig. 2), 211 (64\%) patients received a single antibiotic. Of the total 328 patients, 35\% received ciprofloxacin, 24\% cefazolin, 3\% gentamicin, 1\% TMP-SMX and $1 \%$ nitrofurantoin. A total of $51(16 \%)$ patients received two antibiotics from two separate classes. The most common combinations were $6 \%$ cefazolin with ciprofloxacin, $3 \%$ cefazolin with TMP-SMX, $2 \%$ cefazolin with gentamicin, and $1 \%$ ciprofloxacin with gentamicin. Three patients received antibiotic combinations from three separate classes; 1 patient received ampicillin with gentamicin and ciprofloxacin, and 2 patients received cefazolin with gentamicin and ciprofloxacin. In this population, 63 (19\%) patients received no antibiotic prophylaxis prior to their surgery.

A total of $160(33 \%)$ patients had an indwelling urinary catheter prior to TURP. Of these, 91 (58\%) patients received a single antibiotic regimen. The most common agents prescribed were ciprofloxacin (24\%), cefazolin $(25 \%)$, gentamicin (4\%) and TMP-SMX (3\%) (Table 3, Fig. 3). In total, 50 (30\%) patients received two different antibiotics. The most common combinations were $7 \%$ cefazolin with gentamicin, $2 \%$ cip- rofloxacin with TMP-SMX, 2\% cefazolin and TMP-SMX, and $2 \%$ ciprofloxacin and gentamicin. Eight (5\%) patients received three different antibiotics and 1 patient $(0.6 \%)$ received four different antibiotics. A total of $12(7 \%)$ patients in this group received no antibiotic prophylaxis prior to TURP.

The compliance rate with the AUA BPS in patients without a preoperative catheter was $81 \%$, while the compliance rate for patients with an indwelling catheter prior to TURP was $37 \%$ (Fig. 4).

\section{Discussion}

The use of routine antibiotic prophylaxis prior to TURP is supported by level A evidence and recommended for all patients undergoing this procedure by the AUA's BPS on antimicrobial prophylaxis. ${ }^{1}$ However, there is a paucity of literature describing urologists' prescribing patterns with respect to choice of antibiotic regimen in patients with and without risk factors for infection, particularly in the Canadian healthcare system. In the present study, we assessed an online medical charting system to capture preoperative antibiotics prescribed by urologists for patients undergoing TURP. This study evaluates prescribing practices and compliance with the AUA BPS only; we do not correlate these with outcomes, such as infection rates.

Overall, antibiotic prophylaxis regimens varied significantly, underscoring a lack of consensus with respect to 


\begin{tabular}{|c|c|c|}
\hline Regimen & Antibiotic(s) used & $\begin{array}{c}\text { No. } \\
\text { patients }\end{array}$ \\
\hline \multirow{7}{*}{$\begin{array}{l}\text { Single agent } \\
(n=302)\end{array}$} & Cefazolin & 120 \\
\hline & Ciprofloxacin & 155 \\
\hline & Gentamicin & 16 \\
\hline & TMP-SMX & 6 \\
\hline & Nitrofurantoin & 3 \\
\hline & Norfloxacin & 1 \\
\hline & Cephalexin & 1 \\
\hline \multirow{17}{*}{$\begin{array}{l}\text { Two agents } \\
(n=101)\end{array}$} & Cefazolin + Ciprofloxacin & 41 \\
\hline & Cefazolin + Gentamicin & 18 \\
\hline & Cefazolin + TMP-SMX & 12 \\
\hline & Ciprofloxacin + Gentamicin & 6 \\
\hline & Ciprofloxacin + TMP-SMX & 5 \\
\hline & Ampicillin + Gentamicin & 6 \\
\hline & Cefazolin + Levofloxacin & 1 \\
\hline & Ciprofloxacin + Cephalexin & 1 \\
\hline & Ciprofloxacin + Levofloxacin & 1 \\
\hline & Gentamicin + Nitrofurantoin & 3 \\
\hline & Cefazolin + Flagyl & 1 \\
\hline & Amoxicillin + Clavulin & 1 \\
\hline & Amoxicillin + Cefazolin & 1 \\
\hline & Gentamicin + TMP-SMX & 1 \\
\hline & Ampicillin + Ciprofloxacin & 1 \\
\hline & Tobramycin + Ciprofloxacin & 1 \\
\hline & Piperacillin + Tazobactam & 1 \\
\hline \multirow{5}{*}{$\begin{array}{l}\text { Three agents } \\
(n=11)\end{array}$} & Ampicillin + Gentamicin + Ciprofloxacin & 2 \\
\hline & Cefazolin + Gentamicin + Ciprofloxacin & 5 \\
\hline & Cefazolin + Ciprofloxacin + Ceftriaxone & 1 \\
\hline & Cefazolin + Gentamicin + Nitrofurantoin & 2 \\
\hline & Gentamicin + Ciprofloxacin + TMP-SMX & 1 \\
\hline \multicolumn{2}{|c|}{ No antibacterial prophylaxis } & 74 \\
\hline \multicolumn{2}{|c|}{ Total } & 488 \\
\hline \multicolumn{3}{|c|}{ TURP: transurethral resection of the prostate; TMP-SMX: trimethroprim-sulfamethoxazole. } \\
\hline
\end{tabular}

optimal antibiotic choice prior to this procedure. Moreover, compliance with AUA BPS recommendations was lower than expected, suggesting either a lack of awareness amongst urologists regarding this document, conflicting opinion on its validity or applicability to their practice, and/or error. As the use of prophylactic antibiotics has significant effects on patient outcomes, development of drug resistance and cost to healthcare systems, we believe these results highlight the need for further research, and the development and dissemination of Canadian consensus regarding optimal drug selection prior to this common urological procedure.

There is a lack of evidence to suggest the routine use of one class of antibiotics versus another, with aminoglycosides, flourquinolones, cephalosporins and TMP-SMX all demonstrating efficacy in large meta-analyses. ${ }^{6,18-25}$ This is further supported by a RCT demonstrating equivalent outcomes with the use of levofloxacin versus TMP-SMX as

\begin{tabular}{|c|c|c|}
\hline Regimen & Antibiotic(s) used & $\begin{array}{c}\text { No. } \\
\text { patients }\end{array}$ \\
\hline \multirow{7}{*}{$\begin{array}{l}\text { Single agent } \\
(n=211)\end{array}$} & Cefazolin & 120 \\
\hline & Cefazolin & 80 \\
\hline & Ciprofloxacin & 116 \\
\hline & Gentamicin & 10 \\
\hline & TMP-SMX & 2 \\
\hline & Nitrofurantoin & 2 \\
\hline & Norfloxacin & 1 \\
\hline \multirow{11}{*}{$\begin{array}{l}\text { Two agents } \\
(n=51)\end{array}$} & Cefazolin + Gentamicin & 18 \\
\hline & Cefazolin + Ciprofloxacin & 21 \\
\hline & Cefazolin + Gentamicin & 7 \\
\hline & Cefazolin + TMP-SMX & 9 \\
\hline & Ciprofloxacin + Gentamicin & 3 \\
\hline & Ampicillin + Gentamicin & 5 \\
\hline & Gentamicin + Nitrofurantoin & 2 \\
\hline & Cefazolin + Flagyl & 1 \\
\hline & Ciprofloxacin + TMP-SMX & 1 \\
\hline & Amoxicillin + Clavulin & 1 \\
\hline & Amoxicillin + Cefazolin & 1 \\
\hline \multirow{2}{*}{$\begin{array}{l}\text { Three agents } \\
(n=3)\end{array}$} & Ampicillin + Getamicin + Ciprofloxacin & 1 \\
\hline & Cefazolin + Gentamicin + Ciprofloxacin & 2 \\
\hline \multicolumn{2}{|c|}{ No antibiotic prophylaxis } & 63 \\
\hline \multicolumn{2}{|c|}{ Total } & 328 \\
\hline
\end{tabular}

antibiotic prophylaxis for TURP. ${ }^{2}$ As such, the wide variety of antibiotic regimens used by urologists at our institution is understandable as no single agent stands out as superior with respect to efficacy (Fig. 1). In addition to efficacy, however, the safety, cost, convenience, as well as local bacterial resistance patterns, are considered in the selection of an antibiotic. ${ }^{1}$ While these factors were not included in our data analysis, it is logical that their combination contributed to urologists' antibiotic choice and may explain differences in prescribing patterns. Hence, our results support the initiation of prospective studies to determine the most efficacious antibiotics, as well as the factors that influence urologists' prescribing patterns prior to this procedure.

As no Canadian recommendations exist to guide urologists on optimal antibiotic prophylaxis prior to TURP, we analyzed our practice patterns in reference to recommendations published by the AUA's BPS. We found that compliance with these recommendations was lower than anticipated; $19 \%$ of patients undergoing elective TURP, who did not arrive at the operating room with an indwelling catheter, did not receive preoperative antibiotic prophylaxis (Fig. 4). This suggests that a lack of awareness or acceptance of the BPS may exist, highlighting the need for further research and focused education on this topic. Moreover, as both lack of knowledge and low compliance may be attributed to low 


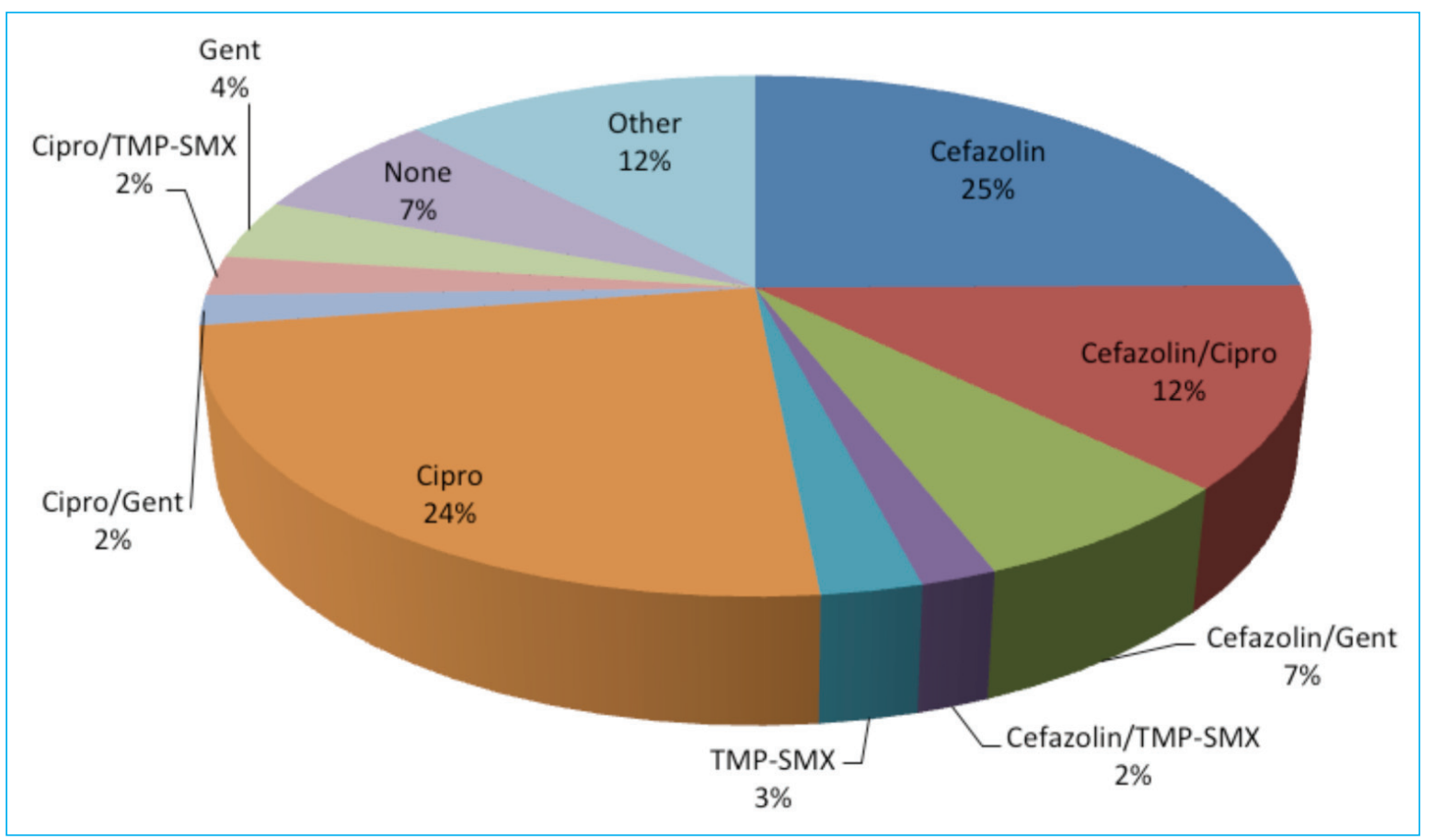

Fig. 3. Pie chart depicting antibacterial prophylaxis for patient with indwelling catheter. Antibiotics given 3 hours preoperatively to 24 hours postoperatively were tabulated. Percentages of total patients with pre-operative indwelling catheters receiving a given antibiotic regimen are depicted. Septra: Septra DS; Cipro: ciprofloxacin; Gent: gentamicin; TMP-SMX: trimethroprim-sulfamethoxazole.

readership of the BPS, we feel that Canadian recommendations may have farther-reaching effects. Alternatively, as no Canadian clinical trials investigating prophylactic antibiotics have been conducted, urologists may question the applicability of the AUA recommendations to our healthcare system. Hence, a lack of faith in the AUA BPS may account for our observed low compliance rates. Future studies will be required to determine this and are of upmost importance in developing successful interventions for changing practice if deemed necessary.

As preoperative catheter status is a significant risk factor for increased post-TURP bacteriuria and infection, ${ }^{26}$ we further characterized prescribing patterns in this patient population. While this has not been previously described, surveys of urologists have highlighted the significantly varied choice of agent used as prophylaxis. ${ }^{27}$ Similarly, we also observed diverse antibiotic regimens in this patient population, again highlighting a lack of consensus among urologists for optimal choice of agent (Fig. 3). The use of gentamicin significantly increased for patients with indwelling catheters, suggesting the preferential use of this agent in this setting; this finding was also consistent with previous studies. ${ }^{27}$

Compliance with the AUA BPS was also quantified for patients with indwelling catheters. We interpret the AUA BPS to suggest that patients with indwelling catheters require two antibiotics from different classes prior to TURP. ${ }^{1}$ Admittedly, this is a subjective interpretation of the BPS, which only states that "additional antibiotic prophylaxis is required for those with risk factors." The use of two antibiotics from two different classes was felt to be appropriate given the increased risk of resistant organism infections in these patients. Using this definition we found that compliance with the BPS was only $37 \%$ (Fig. 4). This is likely at least a slight underestimate, as we could not accurately capture patients who were appropriately treated preoperatively in a targeted manner based on culture and sensitivity results, which the AUA BPS also considers appropriate. If we considered compliance for these patients to be defined in the same way as those without a catheter, it would be calculated at $93 \%$; however, we feel that these patients would have been under-treated.

Along with the reasons for poor compliance listed above, it is also possible, in this setting, that prescribing practice is affected by the often semi-urgent nature of the cases, or the fact that many patients are transferred to the care of an on-call surgeon, creating a gap in preoperative care. Once again, this underscores the necessity of further prospective studies investigating optimal antibiotic choice in this setting, as well as related educational efforts. In the short-term, measures, such as including catheter status and review of cultures in the preoperative briefing, may help raise awareness of the potential need for additional antibiotics in these patients.

The main limitation of this study is its retrospective nature. Additionally, the subjective interpretation of the AUA BPS is also a limitation and may bias our results and underestimate 


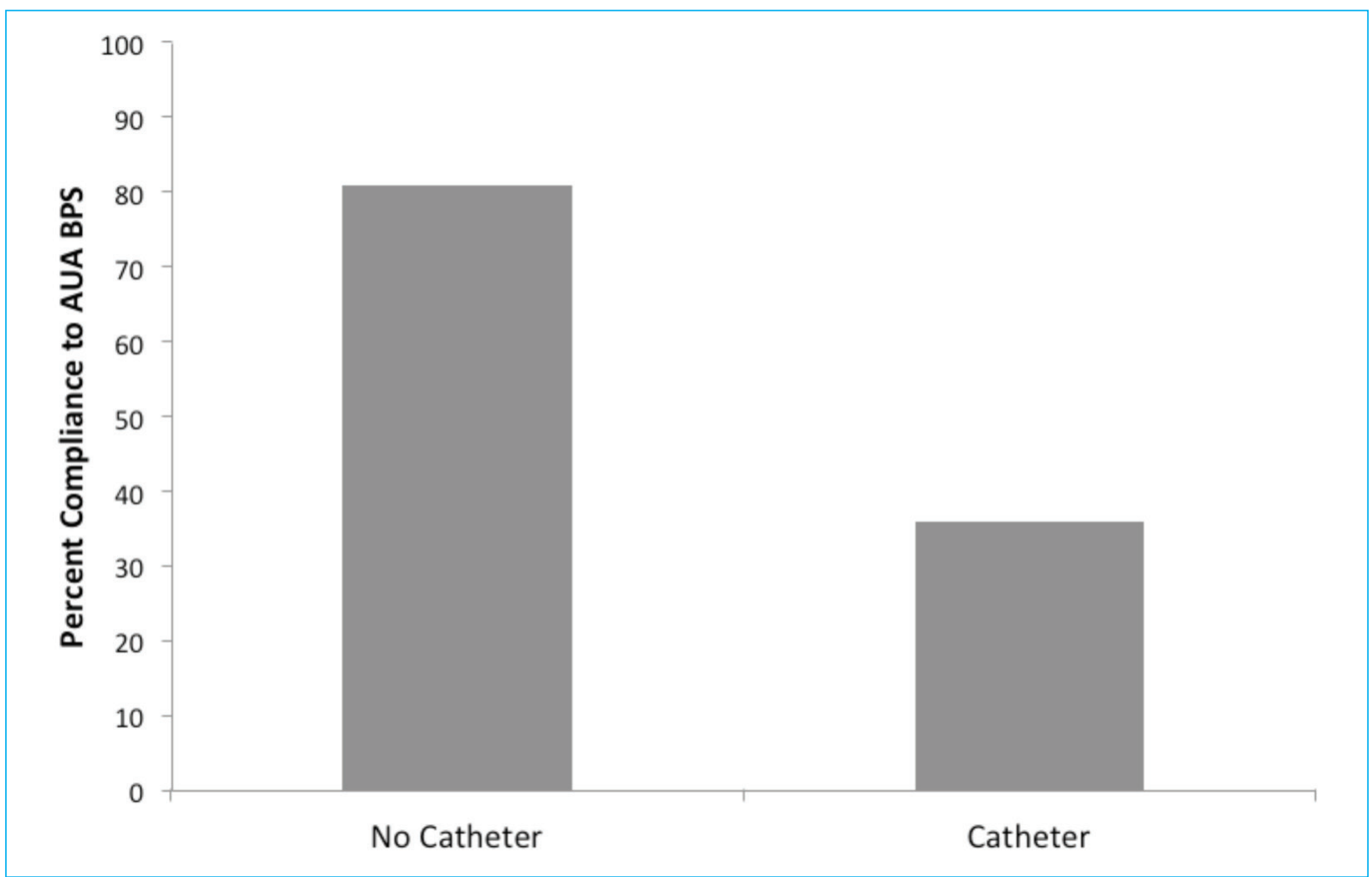

Fig. 4. Compliance rates with AUA BPS. Compliance rates calculated by preoperative catheter status. For patients without a catheter, compliance was defined as those receiving an antibiotic prior to TURP. In patients with an indwelling catheter, compliance was defined as those receiving antibiotics from two different classes prior to surgery. AUA: American Urological Association; TURP: transurethral resection of the prostate; BPS: Best Practice Statement.

compliance rates for patients with indwelling catheters at This paper has been peer-reviewed. the time of TURP.

\section{Conclusion}

Antibiotic prescribing patterns prior to TURP vary considerably, reflecting a lack of consensus among optimal choice of agent for this procedure. While the AUA BPS recommends the routine use of antibiotic prophylaxis for TURP, compliance among urologists is poor, particularly in those patients with indwelling catheters at the time of surgery. Our work highlights the need for further prospective studies investigating optimal choice of antibiotic regimens prior to TURP. Moreover, investigation of those factors contributing to low compliance with AUA guidelines is necessary to determine whether these patterns reflect lack of knowledge among urologists regarding this topic versus inapplicability of the recommendations to our population or healthcare system. Overall, the lack of consensus and compliance highlighted in our study suggests the need for the development of Canadian guidelines.

Competing interests: None declared.

\section{References}

1. Wolf Jr SJ, Bennett CJ, Dmochowski RR, et al. Best Practice Policy Statement on Urologic Surgery Antimicrobial Prophylaxis. J Urol 2008;179:1379-90. http://dx.doi.org/10.1016/i.juro.2008.01.068

2. Wagenlehner FM, Wagenlehner C, Schinzel S, et al. Prospective, randomized, multicentric, open, comparative study on the efficacy of a prophylactic single dose of $500 \mathrm{mg}$ levofloxacin versus $1920 \mathrm{mg}$ trimethoprim/sulfamethoxazole versus a control group in patients undergoing TUR of the prostate. Eur Urol 2005;47:549. http://dx.doi.org/10.1016/j.eururo.2005.01.004

3. Naber KG. Which fluoroquinolones are suitable for the treatment of urinary tract infections? Int J Antimicrob Agents 2001;17:331-41. http://dx.doi.org/10.1016/S0924-8579(00)00362-9

4. Qiang W, Jianchen W, MacDonald R, et al. Antibiotic Prophylaxis for Transurethreal Prostatic Resection in Men with Preoperative Urine Containing Less Than 100,000 Bacteria per ML: A Systematic Review. J Urol 2005:173;1175-81. http://dx.doi.org/10.1097/01.ju.0000149676.15561.cb

5. Goldwasser B. Urinary infections following transurethral resection of bladder tumors. Rate and Source. J Urol 1983:129;1123.

6. Berry A, Barratt A. Prophylactic Antibiotic Use in Transurethral Prostatic Resection: A Meta-Analysis. J Urol 2002:167;571-7. http://dx.doi.org/10.1016/S0022-5347(01)69088-8

7. Brehmer B, Madsen P. Route and prophylaxis of ascending bladder infection in male patients with indwelling catheters. J Urol 1972;108:719.

8. Robinson MR, Arudpragasam ST, Sahgal SM, et al. Bacteremia resulting from prostatic surgery: the source of bacteria. Br J Urol 1982;54:542-6. http://dx.doi.org/10.1111/.1.1464-410X.1982.tb13586.x

9. Cafferkey MT, Falkiner FR, Gillespie WA, et al. Antibiotics for the prevention of septicaemia in urology. J Antimicrob Chemother 1982;9:471-7. http://dx.doi.org/10.1093/jac/9.6.471

10. Madesen PO, Larsen EH, Dorflinger T. Infectious complications after instrumentation of urinary tract. Urology 1985;26:15-7. 
Lawson et al.

\begin{tabular}{|c|c|c|}
\hline Regimen & Antibiotic(s) Used & $\begin{array}{c}\text { No. } \\
\text { patients }\end{array}$ \\
\hline \multirow{6}{*}{$\begin{array}{l}\text { Single agent } \\
(n=91)\end{array}$} & Cefazolin & 120 \\
\hline & Cefazolin & 40 \\
\hline & Ciprofloxacin & 39 \\
\hline & Gentamicin & 6 \\
\hline & TMP-SMX & 4 \\
\hline & Nitrofurantoin & 1 \\
\hline \multirow{14}{*}{$\begin{array}{l}\text { Two agents } \\
(n=50)\end{array}$} & Cephalexin & 1 \\
\hline & Cefazolin + Ciprofloxacin & 20 \\
\hline & Cefazolin + Gentamicin & 11 \\
\hline & Ciprofloxacin + TMP-SMX & 4 \\
\hline & Cefazolin + TMP-SMX & 3 \\
\hline & Ciprofloxacin + Gentamicin & 3 \\
\hline & Cefazolin + Levofloxacin & 1 \\
\hline & Ciprofloxacin + Cephalexin & 1 \\
\hline & Ciprofloxacin + Levofloxacin & 1 \\
\hline & Getnamicin + TMP-SMX & 1 \\
\hline & Gentamicin + Nitrofurantoin & 1 \\
\hline & Ampicillin + Gentamicin & 1 \\
\hline & Ampicillin + Ciprofloxaxacin & 1 \\
\hline & Tobramycin + Ciprofloxacin & 1 \\
\hline \multirow{5}{*}{$\begin{array}{l}\text { Three agents } \\
(n=7)\end{array}$} & Piperacillin + Tazobactam & 1 \\
\hline & Cefazolin + Gentamicin + Ciprofloxacin & 3 \\
\hline & Cefazolin + Ciprofloxacin + Ceftriaxone & 1 \\
\hline & Cefazolin + Gentamicin + Nitrofurantoin & 1 \\
\hline & Getamicin + Ampicillin + Ciprofloxacin & 1 \\
\hline \multicolumn{2}{|c|}{ No antibacterial prophylaxis } & 12 \\
\hline \multicolumn{2}{|c|}{ Total } & 160 \\
\hline
\end{tabular}

11. Grabe M. Antimicrobial agents in transurethral prostatic resection. J Urol 1987:138;245.

12. Simon $H B$, Jeremias $M T$, Greene $L F$, et al. Antibiotic prophylaxis in transurethral prostatic resection. A clinical study. J Urol 195;74:123-8.
13. McEntee GP, McPhail S, Mulvin D, et al. Single dose antibiotic prophylaxis in high risk patients undergoing transurethreal prostatectomy. Br J Surg 1987;74:192. http://dx.doi.org/10.1002/bjs. 1800740312

14. Rodrigues $\mathrm{P}$, Hering $\mathrm{F}$, Meller $\mathrm{A}$, et al. A randomized and prospective study on the value of antibacterial prophylaxis administration in transuretheral resection of prostate. Sao Paulo Med J 2004; 122:4-7. hittp:// dx.doi.org/10.1590/S1516-31802004000100002

15. Sullivan NM, Sutter VL, Mims MM, et al. Clinical aspects of bacteremia after manipulation of the genitourinary tract. J Infect Dis 1973;127:49-55. http://dx.doi.org/10.1093/infdis/127.1.49

16. Schaeffer AJ, Schaeffer EM. Infectious of the urinary tract. In: Campbell-Walsh Urology, 9th ed. AJ Wein, LR Kavoussi, AC Novick, et al. editors. Philadelphia: Saunders-Elsevier;2007:223-303.

17. Colau $A$, Lucet $J C$, Rufat $P$, et al. Incidence and risk factors of bacteriuria after transurethral resection of prostate. Eur Urol 2001;39:272-6. http://dx.doi.org/10.1159/000052452

18. Harvey MH, Leese $\mathrm{T}$, Loyd D, et al. Antibiotic prophylaxis and secondary hemorrhage following transurethral resection of prostate: a prospective trial. Br J Urol 1986;58;450. http://dx.doi.org/10.1111/i.1464410X.1986.tb09102.x

19. Bannister G, Arkell DG, Menday AP. Prostatectomy and prophylaxis. J Antimicrob Chemother 1981;7:209. http://dx.doi.org/10.1093/jac/7.2.209

20. Stricker PD, Grant $A B$. Relative value of antibiotics and catheter care in the prevention of urinary tract infection after transurethral prostatic resection. BrJ Urol 1988;61:494. http://dx.doi.org/10.1111/i.1464410X.1988.tb05087.X

21. Childs SJ, Wells WG, Mirelman S. Antibiotic prophylaxis for genitourinary surgery in community hospitals. J Urol 1983:130;305.

22. Qvist N, Christiansen HM, Ehlers D. Prophylactic antibiotics in transurethral prostatectomy. Urol Res 1984:12;275. http://dx.doi.org/10.1007/BF00258034

23. Cox CE. Comparison of intravenous ciprofloxacin and intravenous cefotaxime for antimicrobial prophylaxis in transurethral surgery. Am J Med 1989:87;252S. hittp://dx.doi.org/10.1016/0002-9343(89)90073-9

24. Gombert ME, duBouchet $L$, Aulicino $T M$, et al. Intravenous ciprofloxacin versus cefotaxime prophylaxis during transurethral surgery. Am J Med 1989:87;250S. hittp://dx.doi.org/10.1016/0002-9343(89)90072-7

25. Christiano AP, Hollowell CM, Kim H, et al. Double-blind randomized comparison of single-dose ciprofloxacin versus intravenous cefazolin in patients undergoing outpatient endourologic surgery. Urology 2000;55:182. http://dx.doi.org/10.1016/S0090-4295(99)00412-4

26. Huang $X$, Shi HB, Wang $X H$, et al. Bacteriuria after bipolar transurethral resection of the prostate: risk factors and correlation with leukocyturia. Urology 2011;77:1183-7. http://dx.doi.org/10.1016/i. urology.2010.08.013

27. Wilson JR, Puri R, Prescott $S$, et al. The catheterized patient undergoing transurethral resection of prostate: a survey of the current practice of British urologists. BJU Int 2003;92:589-91. http://dx.doi. org/10.1046/j.1464-410X.2003.04429.x

Correspondence: Dr. Kevin V. Carlson, Southern Alberta Institute of Urology, Division of Urology, Department of Surgery, University of Calgary, Calgary, AB 